\title{
Expression of matrix metalloproteinase 12 is highly specific for non-proliferating invasive trophoblasts in the first trimester and temporally regulated by oxygen-dependent mechanisms including HIF-1A
}

\author{
Ursula Hiden $^{1}\left({ }^{10} \cdot\right.$ Christian P. Eyth $^{2} \cdot$ Alejandro Majali-Martinez $^{1} \cdot$ Gernot Desoye $^{1}$. \\ Carmen Tam-Amersdorfer ${ }^{2} \cdot$ Berthold Huppertz $^{3} \cdot$ Nassim Ghaffari Tabrizi-Wizsy $^{2}$
}

Accepted: 13 September 2017 / Published online: 9 October 2017

(C) The Author(s) 2017. This article is an open access publication

\begin{abstract}
During first trimester pregnancy, trophoblast cells invade from the placenta into the maternal decidua where they anchor the placenta and remodel luminal structures like spiral arteries. This process depends on proteases secreted by invading trophoblasts, which degrade extracellular matrix (ECM). We here aimed to identify proteases particularly important for trophoblast invasion. We generated a list of proteases capable of degrading decidual ECM and trophoblast integrins using MEROPS database and compared expression of these proteases between primary trophoblasts isolated from first trimester placenta (FT, $n=3$ ), representing an invasive phenotype, vs trophoblasts isolated from term pregnancy (TT, $n=3$ ), representing a non-invasive trophoblast phenotype. Matrix metalloproteinase 12 (MMP12) revealed highest expression levels in FT, with absent expression in TT. In situ hybridisation and immunofluorescence localised MMP12 specifically to extravillous trophoblasts (evCT) whilst Ki67 co-staining revealed that proliferating trophoblasts of the cell columns were almost negative for MMP12. Quantification revealed a decline in MMP12 positive evCT at the end of first trimester,
\end{abstract}

Electronic supplementary material The online version of this article (doi:10.1007/s00418-017-1608-y) contains supplementary material, which is available to authorized users.

Ursula Hiden

ursula.hiden@medunigraz.at

1 Department of Obstetrics and Gynecology, Medical University of Graz, Graz, Austria

2 Institute of Pathophysiology and Immunology, Center of Molecular Medicine, Medical University of Graz, Graz, Austria

3 Institute of Cell Biology, Histology and Embryology, Medical University of Graz, Graz, Austria when oxygen levels start rising. MMP12 promoter analysis identified potential binding sites for hypoxia-inducible factor (HIF-1) and other oxygen-sensitive transcription factors. Moreover, MMP12 protein was increased by low oxygen in FT in vitro and by addition of a HIF- $1 \alpha$ activator. Collectively, MMP12 is a highly expressed protease specific for invasive evCT during the first trimester. MMP12 down regulation by increasing oxygen concentration enables temporal expression control of MMP12 and involves several mechanisms including HIF-1 $\alpha$. These findings suggest MMP12 involved in trophoblast invasion during the first trimester.

Keywords Matrix metalloproteinase $12 \cdot$ Trophoblast invasion $\cdot$ HIF- $1 \alpha \cdot$ Hypoxia

\section{Introduction}

During the first trimester of pregnancy, a specific subset of placental trophoblasts differentiate into invasive, extravillous cytotrophoblasts (evCT) and invade into the maternal decidua. Along their invasion pathway, evCT form compact cell columns that anchor the placenta to the uterine wall. A number of evCT invade further towards luminal structures within the placental bed (Moser and Huppertz 2017), such as the uterine spiral arteries, which are remodelled into low resistance vessels. Trophoblast invasion depends on the function of proteases that are capable to degrade extracellular matrix (ECM) components (Ghaffari-Tabrizi-Wizsy et al. 2014; Cohen et al. 2006; Anacker et al. 2011) present in the decidua, i.e. various collagens, laminin, fibronectin, fibrillin and vitronectin (Kemp et al. 2002; Oefner et al. 2015; Aplin et al. 2000). Furthermore, before evCT can start to move and migrate through the degraded ECM along their 
invasion pathway, evCT need to detach their connections to the ECM and thus, cleave integrins through which they adhere (Kemp et al. 2002). In fact, various proteases were identified in invading trophoblasts in the first trimester, which participate in the process of invasion. For instance, matrix metalloproteinases (MMPs) are a family of key proteinases involved in ECM degradation, and various MMPs including MMP2, 9, 7 and 14 were implicated in trophoblast invasion in vitro and in vivo (reviewed by (Cohen et al. 2006)). Besides MMPs, also other proteases contribute to ECM degradation or to integrin shedding to eventually facilitate trophoblast invasion, such as members of the ADAMs (a disintegrin and metalloproteinase) family (Pollheimer et al. 2014).

Trophoblast invasion is tightly regulated by multiple factors including cytokines, hormones and growth factors (Bischof and Irminger-Finger 2005). However, oxygen is one of the most potent regulators (Huppertz et al. 2009; Pringle et al. 2010): Whilst a population of invasive trophoblasts modifies and widens the uterine spiral arteries in the first weeks of gestation, some trophoblasts form plugs to prevent immediate maternal blood flow to the placenta, thus creating a low oxygen environment within the placenta. Only at the end of the first trimester do plugs start to detach and placental oxygen levels rise (Kaufmann et al. 2003; Jauniaux et al. 2000). This rise in oxygen is an important signal for foeto-placental development and limits trophoblast invasion. Concomitantly, expression of various proteases involved in the invasion process decreases (Xu et al. 2000).

Our aim was to identify proteases which are particularly important for trophoblast invasion. For this purpose we generated a list of proteases that degrade ECM components present in the decidua and integrins expressed by invasive trophoblasts and compared these proteases between isolated primary trophoblasts from first trimester placenta (FT), representing an invasive, mostly HLA-G positive phenotype (GhaffariTabrizi-Wizsy et al. 2014), and trophoblasts from term placenta (TT), representing a non-invasive trophoblast population (Hohn and Denker 2002; Shimonovitz et al. 1994). Among such potentially invasion-relevant proteases, the protease with highest expression levels in isolated first trimester trophoblasts was MMP12, with absent expression in trophoblasts at term. Therefore, we further investigated placental gene and protein expression patterns of MMP12 throughout the first trimester, explored a possible role of oxygen in its regulation and thus, identified MMP12 as a new, spatio-temporally regulated protease expressed by invasive, evCT of the cell columns.

\section{Materials and methods}

\section{Ethics statement}

The study was approved by the Ethics Committee of the Medical University of Graz. All women gave written informed consent for collection and investigational use of tissues.

\section{Tissue collection}

Placental villi and decidua from first trimester were obtained from elective vaginal terminations of first-trimester pregnancies (gestational age 6-11 weeks) $(n=23)$. For immunohistochemistry, immunofluorescence and in situ hybridisation, tissues were fixed overnight in $4 \%$ paraformaldehyde at $4{ }^{\circ} \mathrm{C}$ before processing to paraffin blocks. Primary trophoblasts were isolated from washed placental villi.

\section{Isolation of trophoblasts}

First trimester trophoblasts $(n=17)$ were isolated by enzymatic digestion with trypsin/dispase (Gibco, UK/Roche, Germany), Percoll (Sigma, USA) centrifugation and negative magnetic bead (Dynabeads, Thermo Fisher, Norway) immunopurification with the anti-leukocyte marker CD45 (Invitrogen, Norway) and anti-fibroblast marker CD90 (Dianova, Germany), as described (Blaschitz et al. 2000). Isolated cells were resuspended in DMEM (Gibco) supplemented with $10 \%$ FBS. Term trophoblasts $(n=10)$ were isolated from third trimester placenta as described (Cervar et al. 1999). Briefly, minced villous tissue was digested with a trypsin/dispase/DNase (Sigma) solution. After Percollgradient centrifugation a negative selection with magnetic beads conjugated to MCA-81 (Sigma) was performed. Isolated villous trophoblasts were cultured in DMEM supplemented with $10 \%$ FBS. First trimester and term trophoblasts were tested for viability and differentiation by measuring $\beta$-human chorionic gonadotropin secretion (Dade Behring, USA) (Polliotti et al. 1995). Purity was confirmed by immunocytochemical staining for the trophoblast marker cytokeratin 7 (CK7, Dako, Denmark) and for the mesenchymal cell marker vimentin (Dako) (Cervar et al. 1999). Only preparations of $\geq 99 \%$ purity were used. For gene expression analysis, first trimester $(n=10)$ and term trophoblasts $(n=10)$ were cultured at $37^{\circ} \mathrm{C}$ and $21 \%$ oxygen for $24 \mathrm{~h}$. For Immunoblotting, first trimester trophoblasts $(n=7)$ were cultured at $37^{\circ} \mathrm{C}$ and 5,12 and $21 \%$ oxygen for $48 \mathrm{~h}$.

Primary trophoblasts isolated either from first trimester placenta from week 7 to 10 of pregnancy (GEO accession No GSE59126) or from term placenta (GEO Accession No GSE69086) were subjected to gene expression analysis using HU-133A GeneChips (Affymetrix, Santa Clara, CA). 
RNA was isolated with Trizol (MRC, Cincinnati, OH, USA) followed by quality assessment using a bioanalyser (Agilent, Palo Alto, CA, USA). For first trimester trophoblasts as well as for term trophoblast, 3 microarrays were performed. On each microarray, total RNA from 5 cell preparations (first trimester trophoblast: GEO Accession Number GSE59126; term trophoblast: GEO Accession Number GSE69086), isolated from different placentas, was pooled. cDNA was synthesised from $5 \mu \mathrm{g}$ of pooled RNA (SuperScript DoubleStranded cDNA Synthesis Kit; Invitrogen, Carlsbad, USA), transcribed in vitro (RNA Transcript Labeling Kit; Enzo diagnostics, Farmingdale, NY, USA) and fragmented. To test the quality of the cRNA, it was hybridised against test-3 arrays (Affymetrix). As samples passed the quality criteria (bioC, bioD and cre were present, the $3^{\prime}: 5^{\prime}$ ratio of the polyA controls was $<3$ ), the cRNAs were hybridised against Affymetrix HU133A chips. RNA preparation and hybridisation followed the Affymetrix user manual. Data analysis of raw data was normalised globally and processed with Microarray Suite, version 5.0 (Affymetrix) and Data Mining Tool (Affymetrix) software (Huppertz et al. 2009). Genes were classified as being expressed if signal intensity $>200$. Annotations were obtained from NetAffx (available at http://www. affymetrix.com, last accessed in December 2013).

For protease analysis, we defined proteases as potentially invasion relevant if they cleaved either decidual ECM components (collagens I, III, IV, V, VI, laminins, fibronectin, vitronectin) or trophoblast integrins (integrins $\alpha 6 \beta 4, \alpha 5 \beta 1$ and $\alpha v \beta 3$ ). Using MEROPS database (http://merops.sanger. ac.uk/, release 11.0 (2017)) (Rawlings et al. 2016) we generated a list of 53 proteases capable of cleaving these substrates, of which 46 were analysed by the microarray.

\section{Semi-quantitative RT-PCR}

Total RNA was isolated from isolated trophoblasts from first trimester $(n=5)$ and term placenta $(n=5)$ using the TRI reagent RT kit (Molecular Research Center Inc., Cincinnati, OH, USA) according to the manufacturer's instructions. Quality and quantity of the RNA were proven utilising the NanoDrop ND-1000 spectrophotometer (PEQLAB Biotechnology $\mathrm{GmbH}$, Erlangen, Germany) and ethidium bromide stained $1 \%$ agarose gel electrophoresis. For reverse transcription and amplification, the Qiagen one-step PCR kit (Qiagen, Hilden, Germany) was used on a PTC-200 Thermocycler (GMI Inc., Ramsey, MI, USA) using the preinstalled RT27 program, which consists of three steps starting with the reverse transcription at $50{ }^{\circ} \mathrm{C}(30 \mathrm{~min})$ and an initial PCR activation step at $95^{\circ} \mathrm{C}(15 \mathrm{~min})$. The second step includes 27 cycles of denaturation at $94{ }^{\circ} \mathrm{C}(30 \mathrm{~s})$, annealing at $60{ }^{\circ} \mathrm{C}$ (30 s) and extension with a temperature of $72{ }^{\circ} \mathrm{C}(60 \mathrm{~s})$ followed by the final extension at $72{ }^{\circ} \mathrm{C}(10 \mathrm{~min})$ in the third step. The gene encoding ribosomal protein L30 (RPL30) was used as a housekeeping gene. The primer pair CACCTG ACATGAACCGTGA/GCAGAGAGGCGAAATGTGT was used for amplification of MMP12 and CCTAAGGCAGGA AGATGGTG/CAGTCTGTTCTGGCATGCTT for RPL30. Quality assessment was performed on an ethidium bromide stained $1.5 \%$ agarose gel, visualised and documented by the ChemiDoc XRS transluminometer (Bio-Rad, Germany).

\section{Quantitative RT-PCR}

Complementary DNA (cDNA) was synthesised with the High Capacity cDNA Reverse Transcription Kit (AB Life Tech Austria, Vienna) using random hexamer primer with the following protocol: $10 \mathrm{~min}$ at $25^{\circ} \mathrm{C}, 120 \mathrm{~min}$ at $37^{\circ} \mathrm{C}$ for reverse transcription and a 5 -s step at $85{ }^{\circ} \mathrm{C}$ for enzyme inactivation. The cDNA was diluted 1:20 with nuclease-free water before performing the PCR. For the mastermix $4 \mu \mathrm{l}$ of $5 \mathrm{ng} / \mu \mathrm{l}$ diluted cDNA, $7.5 \mu \mathrm{l}$ iQ SybrGreen Supermix (Bio-Rad), $0.2 \mu \mathrm{M}$ forward and reverse primer was used and filled up to a total volume of $15 \mu \mathrm{l}$ with nuclease-free water. All samples were run in triplicate. Expression of MMP12 (forward primer CCACTGCTTCTGGAGCTCTT, reverse primer TCTCGTGAACAGCAGTGAGG) and of the house keeping gene HPRTl (forward primer GACCAGTCAACA GGGGACAT, reverse primer CTGCATTGTTTTGCCAGT GT) was measured with CFX96 from BioRad under the following conditions: $95^{\circ} \mathrm{C}$ for $3 \mathrm{~min}$, followed by 40 cycles of $95^{\circ} \mathrm{C}$ for $10 \mathrm{~s}, 60^{\circ} \mathrm{C}$ for $30 \mathrm{~s}$ and $72^{\circ} \mathrm{C}$ for $10 \mathrm{~s}$. This was ensued by $30 \mathrm{~s}$ at $95^{\circ} \mathrm{C}$ and $30 \mathrm{~s}$ at $55^{\circ} \mathrm{C}$ followed by a melt curve analysis from 55 to $95{ }^{\circ} \mathrm{C}$ in $0.5^{\circ} \mathrm{C}$ steps lasting $5 \mathrm{~s}$. Data were normalised to HPRT1 and relative gene expression was assessed using the $2^{-\Delta \Delta \mathrm{Ct}}$-method.

\section{Radioactive in situ hybridisation}

Riboprobes were synthesised by in vitro transcription of linearized pDrive plasmid templates containing the appropriate insert, using T7 or SP6 RNA polymerase (Promega) and ${ }^{35}$ S-labelled UTP (NEN/Perkin Elmer) to generate antisense and sense probes. Tissues sections were deparaffinized, fixed in $4 \%$ paraformaldehyde and treated with proteinase $\mathrm{K}$. After washing in $0.5 \times \mathrm{SSC}$, the sections were covered with hybridisation solution (50\% deionised formamide, $0.3 \mathrm{M} \mathrm{NaCl}, 20 \mathrm{mM}$ Tris (pH 8.0), 5.0 mM EDTA, $1 \times$ Denhardt's solution, $10 \%$ Dextran Sulphate, and $10 \mathrm{mM}$ DTT) and prehybridised for $2 \mathrm{~h}$ at $55{ }^{\circ} \mathrm{C} .{ }^{35} \mathrm{~S}$-labelled antisense and sense RNA probes $\left(3 \times 10^{5} \mathrm{cpm} /\right.$ slides $)$ were added to the hybridisation solution, and the incubation continued for $12-18 \mathrm{~h}$ at $57^{\circ} \mathrm{C}$. After hybridisation, the sections were washed for $20 \mathrm{~min}$ in $2 \mathrm{X} \mathrm{SSC}, 10 \mathrm{mM} \beta$-mercaptoethanol and $1 \mathrm{mM}$ EDTA, treated with RNAse A $(10 \mu \mathrm{g} / \mathrm{ml})$ for 
$30 \mathrm{~min}$ at room temperature and washed at high stringency (0.1 x SSC, $10 \mathrm{mM} \beta$-mercaptoethanol, $1 \mathrm{mM}$ EDTA) for $2 \mathrm{~h}$ at $60{ }^{\circ} \mathrm{C} ; 20 \mathrm{~min}$ in $0.5 \times \mathrm{SSC}$. Tissue sections were then dehydrated in graded ethanol containing $0.3 \mathrm{M}$ ammonium acetate, air dried, and dipped in photographic emulsion NTB-2 (Kodak). Slides were stored at $4{ }^{\circ} \mathrm{C}$ in light-tight boxes; after 6 weeks' exposure sections were developed and counterstained with haematoxylin and eosin.

\section{DIG in situ hybridisation}

Digoxigenin-11-UTP Labeling Kit with T7 and SP6 RNA polymerases was used (Roche Diagnostics Corp. Indianapolis, USA) to generate antisense and sense probes. Briefly, tissue sections were deparaffinised, fixed in $4 \%$ paraformaldehyde and treated with proteinase K. After washing in $0.5 \times \mathrm{SSC}$, the sections were covered with $100 \mathrm{ml}$ of hybridisation solution (50\% deionized formamide, $0.3 \mathrm{M}$ $\mathrm{NaCl}, 20 \mathrm{mM}$ Tris (pH 8.0), 5.0 mM EDTA, $1 \times$ Denhardt's solution, $10 \%$ Dextran sulfate, and $10 \mathrm{mM}$ DTT) and prehybridised for $30 \mathrm{~min}$ at $55{ }^{\circ} \mathrm{C}$. Excess liquid was drained off before $100 \mu \mathrm{l}$ of probe was added and the incubation continued for $12-18 \mathrm{~h}$ at $57^{\circ} \mathrm{C}$. After hybridisation, the sections were washed for $20 \mathrm{~min}$ in $2 \times \mathrm{SSC}$ and $1 \mathrm{mM}$ EDTA, treated with RNAse A $(10 \mu \mathrm{g} / \mathrm{ml})$ for $30 \mathrm{~min}$ at room temperature and washed at high stringency $(0.1 \times \mathrm{SSC}, 1 \mathrm{mM}$ EDTA) for $2 \mathrm{~h}$ at $60^{\circ} \mathrm{C} ; 20 \mathrm{~min}$ in $0.5 \times \mathrm{SSC}$. Hybridised probe was detected using an Anti-Digoxigenin-AP, Fab fragments (Roche and NBT-BCIP colour substrate mix (Roche). Sections were counter stained with $0.1 \%(\mathrm{w} / \mathrm{v})$ methyl green (Sigma).

\section{Immunofluorescence}

Studies were carried out on 4\% PFA-fixed, paraffin-embedded placental villous and decidual tissues of first trimester and term placenta, sectioned at $5 \mu \mathrm{m}$. Slides were deparaffinised in xylene and rehydrated with decreasing concentrations of ethanol according to standard methods. Antigen retrieval was used for tissue sections submerged in $10 \mathrm{mM}$ Sodium Citrate Buffer, 0.05\% Tween-20 (pH 6.0) for $10 \mathrm{~min}$ in a domestic microwave oven. Slides were allowed to cool down for $45 \mathrm{~min}$ at room temperature before rinsing in wash buffer Tris-Buffered Saline Tween-20 (TBST, pH 7.4). Sections were blocked with UV ultra block for 7 min before primary antibody incubation at $4{ }^{\circ} \mathrm{C}$ overnight. In all double stainings, rabbit anti-human MMP-12 antibody was used to stain MMP12 (0.67 $\mu \mathrm{g} / \mathrm{ml}$, ab38935, Abcam, Cambridge, MA). Sections were costained for HLA-G, a marker for extravillous trophoblasts (mouse anti human HLA-G clone MEM-G/1, $0.5 \mu \mathrm{g} / \mathrm{ml}$, BioVendor, Heidelberg, Germany), for cytokeratin 7, a marker for all trophoblast subpopulations (mouse anti-human cytokeratin-7 clone PG-M1, $0.21 \mu \mathrm{g} / \mathrm{ml}$,
Dako Cytomation) and for Ki67, a marker for proliferating cells (mouse anti human Ki67 clone MIB-1, $1.07 \mu \mathrm{g} /$ ml, Dako Cytomation). Negative controls were incubated with the appropriate IgG fractions as isotype controls. All incubation steps were performed in a dark moist chamber at room temperature. After 5 min of TBST wash, secondary antibodies (goat anti-rabbit labelled with Dylight 488 and goat anti-mouse conjugated with Cy3, diluted 1:800, Jackson ImmunoResearch Laboratories, West Grove, PA) were applied for $30 \mathrm{~min}$. Followed by another TBST wash, DAPI (blue) $5 \mathrm{mg} / \mathrm{ml}$ was added to the slides for $20 \mathrm{~min}$ as a nuclei counter stain. The sections were rinsed again with TBST before mounting with Vectashield mounting medium (Vector Lab, Inc., Burlingame, CA, USA). Images were acquired on a Leica DM4000 B trinocular microscope equipped with a Leica DFC300 FX digital camera and processed with the image capturing software Leica Application Suit, Version 3.6.0 (all three: Leica Microsystems Ltd., Heerbrugg, Switzerland).

\section{Quantification of MMP12 positive cells}

HLA-G positive cells and MMP12 positive cells within the cell columns were quantified. Only cell columns that were entirely cross sectioned (from the villus to the distal part of the column) on the slide were used for analysis and tagged using GIMP (Open Source, GNU General Public License, Version 3) and subsequently counted by Motic Images Advanced 3.2 (Motic Deutschland GmbH, Germany). Data were analysed in Microsoft Excel (Microsoft, Redmond, WA) by calculating the ratio of MMP12 positive cells among the HLA-G positive cells for each cell column.

\section{Promoter analysis}

The $M M P 12$ promoter was analysed for potential transcription factor binding sites using PROMO bioinformatics tool (Version 3.0.2) at http://alggen.lsi.upc.es/cgi-bin/promo_v3/ promo/promoinit.cgi?dirDB=TF_8.3. MMP12 promoter sequence (2000 bp upstream of the transcriptional) was obtained from UCSC Genome Browser (https://genome. ucsc.edu/). The maximum matrix dissimilarity rate was restricted to $2 \%$ and the Random Expectation query, which gives the number of expected occurrences of the match in a random sequence, was restricted to $<0.2$.

\section{Immunoblotting}

Immediately after isolation, primary first trimester trophoblasts from 4 different placentas, each in triplicate, were cultured at 5, 12 and $21 \%$ oxygen for $48 \mathrm{~h}$. With an additional set of experiments, a further treatment with the HIF- $1 \alpha$ activator Desferrioxamine (DFO, Sigma) was performed at 
$21 \%$ oxygen. DFO is an iron chelator that prevents HIF- $1 \alpha$ degradation and thus, promotes HIF-1 action (Salceda and Caro 1997). To test the optimal DFO concentration, 10, 50 and $100 \mu \mathrm{M}$ DFO was added. Since $100 \mu \mathrm{M}$ gave the best results, this concentration was chosen for the experiments. DFO treatment was performed in primary first trimester trophoblasts from three different placentas, each in triplicate. For Western blotting, lysates of total cell proteins were prepared in RIPA buffer (Sigma) containing protease inhibitors (Roche). Protein concentration was determined by the bicinchoninic acid assay (BCA). Equal amounts of protein were mixed with Laemmli buffer (Sigma) and denatured at $96{ }^{\circ} \mathrm{C}$ for $5 \mathrm{~min}$. Samples were loaded onto 10\% SDS-PAGE gels (Bio-Rad), resolved at $140 \mathrm{~V}$ for $1 \mathrm{~h}$ and transferred to nitrocellulose membranes (Bio-Rad). Non-specific binding sites were blocked for $1 \mathrm{~h}$ with $5 \%$ non-fat dry milk (Bio-Rad) in tris-buffered saline (TBS) $+0.1 \%$ Tween 20 (Sigma). After blocking, membranes were incubated with anti MMP12 antibody (Abcam, 1:1000) and or $\beta$-actin (Abcam, 1:25,000) overnight at $4{ }^{\circ} \mathrm{C}$. Blots were subsequently washed and incubated with the HRP-conjugated secondary antibody (goat anti rabbit, Bio-Rad Laboratories, 1:2000 for MMP12 and 1:25,000 for $\beta$-actin). Immunolabeling was detected using SuperSignal West Pico Chemiluminescent Substrate (Thermo Scientific) and visualised with the Chemidoc XRS software (Bio-Rad). Band density was quantified using the Alpha Digidoc software (Alpha Innotech Corp, Innsbruck, Austria).

\section{Statistical evaluation}

Kruskal-Wallis test was used to compare the ratio of MMP12 positive cells in the different gestational weeks and to analyse the oxygen effect on MMP12 levels and for pairwise analysis, Dunn's Multiple Comparison Test as post test for pairwise analysis.

\section{Results}

\section{MMP12 expression is specific for first trimester trophoblasts}

In order to identify proteases specifically important for trophoblast invasion, we compared gene expression of potentially invasion relevant proteases between primary FT, representing an invasive phenotype paralleled by expression of HLA-G (Ghaffari-Tabrizi-Wizsy et al. 2014), with TT, representing a non-invasive phenotype. Proteases were selected according to their ability to degrade major ECM components of the decidua, i.e. collagens I, III, IV, V and VI, laminin, fibronectin, fibrillin 1 and 2 and vitronectin (Kemp et al. 2002; Oefner et al. 2015; Aplin et al. 2000). Furthermore, we included proteases capable to cleave integrins expressed by invasive trophoblasts, i.e. integrins $\alpha 6 \beta 4, \alpha 5 \beta 1$ and $\alpha v \beta 3$ (Kemp et al. 2002). Using MEROPS database, we identified 53 human proteases involved in cleavage of these substrates, of which 46 were measured by the microarray (Online Resource 1). 24 of these proteases were expressed in FT with 12 of them being higher expressed in FT than in noninvasive TT. These were regarded as potentially invasion relevant. The proteases with highest expression level in FT and with largest expression difference (84-fold) between FT and TT were MMP12 (Fig. 1a). Gene expression of MMP12 was confirmed by semi-quantitative RT-PCR (Fig. 1b) and by quantitative RT-PCR (not shown). Besides MMP12, also other MMPs (MMP2, 15, 3, 14, 7 and 9) and ADAMs (ADAM12 and ADAMTS5) were identified by the screening, as well as PLAU (urokinase-type plasminogen activator) and the two serine proteases PCSK6 and 7 (proprotein convertase subtilisin/kexin type-6 and -7).

\section{MMP12 expression is specific for evCT}

Localisation of MMP12 mRNA and protein in first trimester placental tissues using in situ hybridisation and immunofluorescence confirmed the potential role of MMP12 in trophoblast invasion. In situ hybridisation was performed using two different techniques, i.e. radioactive vs DIG labelled probes. For immunofluorescence, sections were double stained for HLA-G, a marker for evCT (Moser et al. 2011). All methods revealed positive MMP12 mRNA and protein expression in a specific subset of the evCT of the cell columns The MMP12 antibody revealed also a faint signal in the syncytiotrophoblast, in villous cytotrophoblasts and in some stromal cells. However, since both in situ hybridisation methods gave no signal for MMP12 mRNA in these compartments, limited production of MMP12 protein by these cells may be possible but precarious (Fig. 2a-d). Moreover, as described previously (Harris et al. 2010), MMP12 was present in the evCT invading and remodelling the uterine vessels, i.e. endovascular trophoblasts (not shown). Within the cell columns, MMP12 expression was primarily located to a distinct, scattered subpopulation. In order to identify whether MMP12 production may be specific for proliferating cells, sections were co-stained for the proliferation marker Ki67. The proliferative trophoblasts of the proximal part of the cell columns were stained for Ki67, but only few cells were positive for MMP12, whilst the invasive evCT of the distal part of the columns produced MMP12, but were mostly negative for Ki67 (Fig. 3a, b).

\section{Temporal changes in expression of MMP12 by evCT}

In order to determine whether MMP12 expression during the first trimester is temporally regulated, we quantified MMP12 
Fig. 1 (a) Proteases capable to degrade decidual ECM and integrins, expressed by primary trophoblasts from first trimester (FT) and term of gestation (TT). Among all proteases degrading collagens I, III, IV, $\mathrm{V}$ and VI, laminin, fibronectin, fibrillin 1 and 2, vitronectin and integrins specific for extravillous trophoblasts, MMP12 was highest expressed in FT with almost absent expression in TT. MMPs are coloured in black. Data are shown as mean \pm SD. b The expression difference of $M M P 12$ in primary first trimester (FT) vs. term trophoblasts (TT) was confirmed by semiquantitative RT-PCR using RPL3O as housekeeping gene $\mathbf{a}$

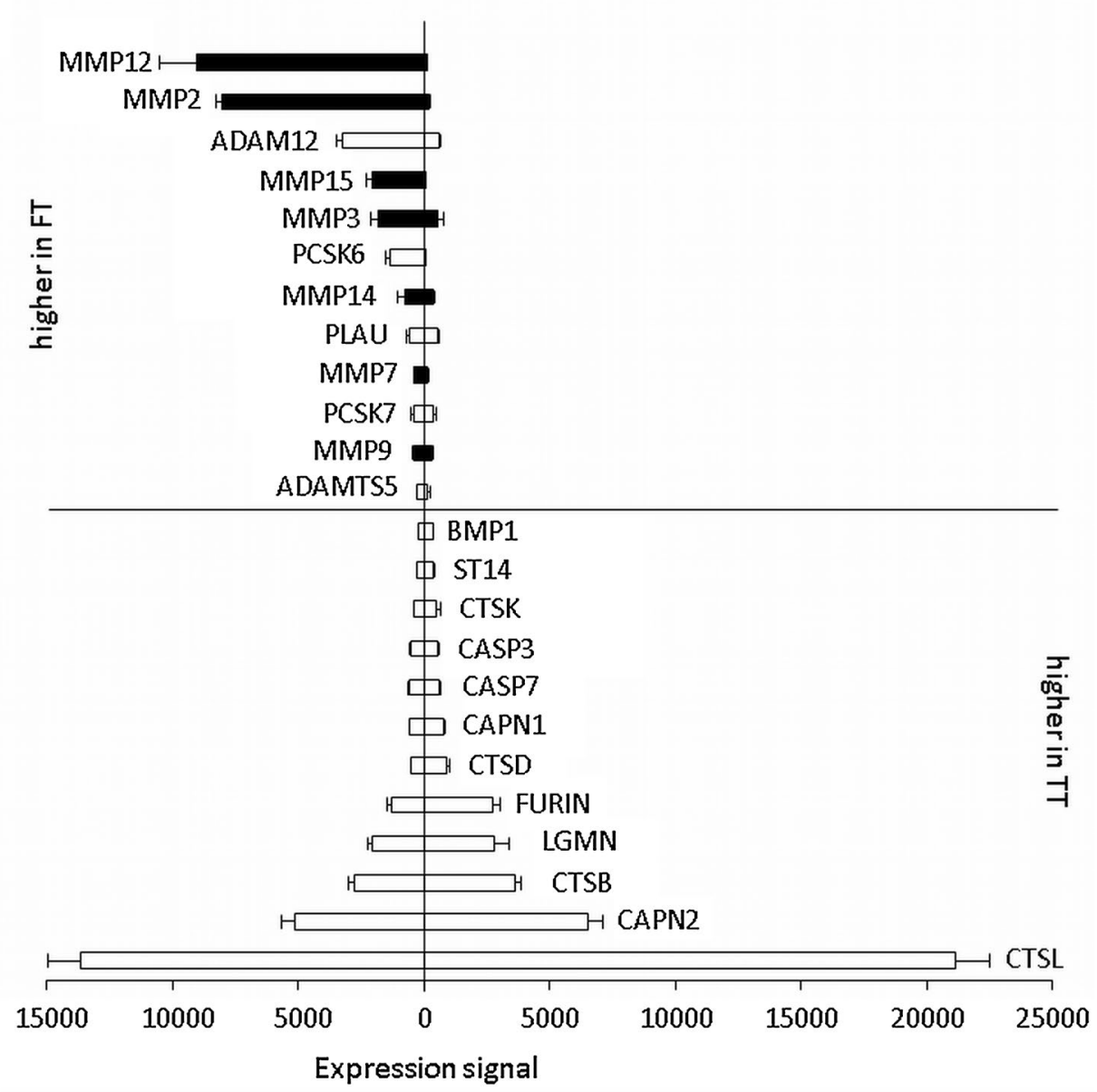

b

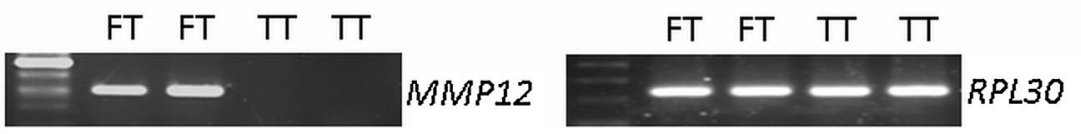

positive cells in placental specimen of different gestational weeks, i.e. in weeks 6-7, 8-9 and 10-11, using image analysis of HLA-G and MMP12 double-labelled cells (Online Resource 2). There was a significant reduction in the ratio of MMP12 positive cells within all evCT towards the end of the first trimester, i.e. in weeks 10-11 vs earlier weeks (Fig. 4).

\section{MMP12 expression is regulated by oxygen}

To determine potential regulators of MMP12 expression, a transcription factor binding site (TFBS) analysis of the MMP12 promoter using PROMO bioinformatics tool (Messeguer et al. 2002; Farre et al. 2003; Fekry et al. 2016) was performed. The analysis revealed TFBS already described in the MMP12 promoter, such as AP-1, wnt signalling and PEA3 binding sites, but in addition furthermore identified other potential TFBS, including a HIF-1 binding site (Online Resource 3). Thus, several binding sites for hypoxia-sensitive transcription factors exist in the MMP12 promoter, including HIF-1. Therefore, we investigated the impact of oxygen on MMP12 regulation and measured MMP12 protein in primary FT cultured at different oxygen concentrations. Immunoblotting demonstrated that higher levels of oxygen significantly reduced MMP12 when compared to $5 \%$ oxygen (Fig. 5). Concordantly, activation of HIF- $1 \alpha$ with its specific activator DFO increased MMP12 level even in the presence of $21 \%$ oxygen (Fig. 5).

\section{Discussion}

Trophoblast invasion depends on proteases. We here aimed to identify proteases potentially relevant for this fundamental process in pregnancy establishment. Gene expression analysis identified MMP12 as a proteolytic enzyme highly expressed in primary FT that might be capable of degrading 

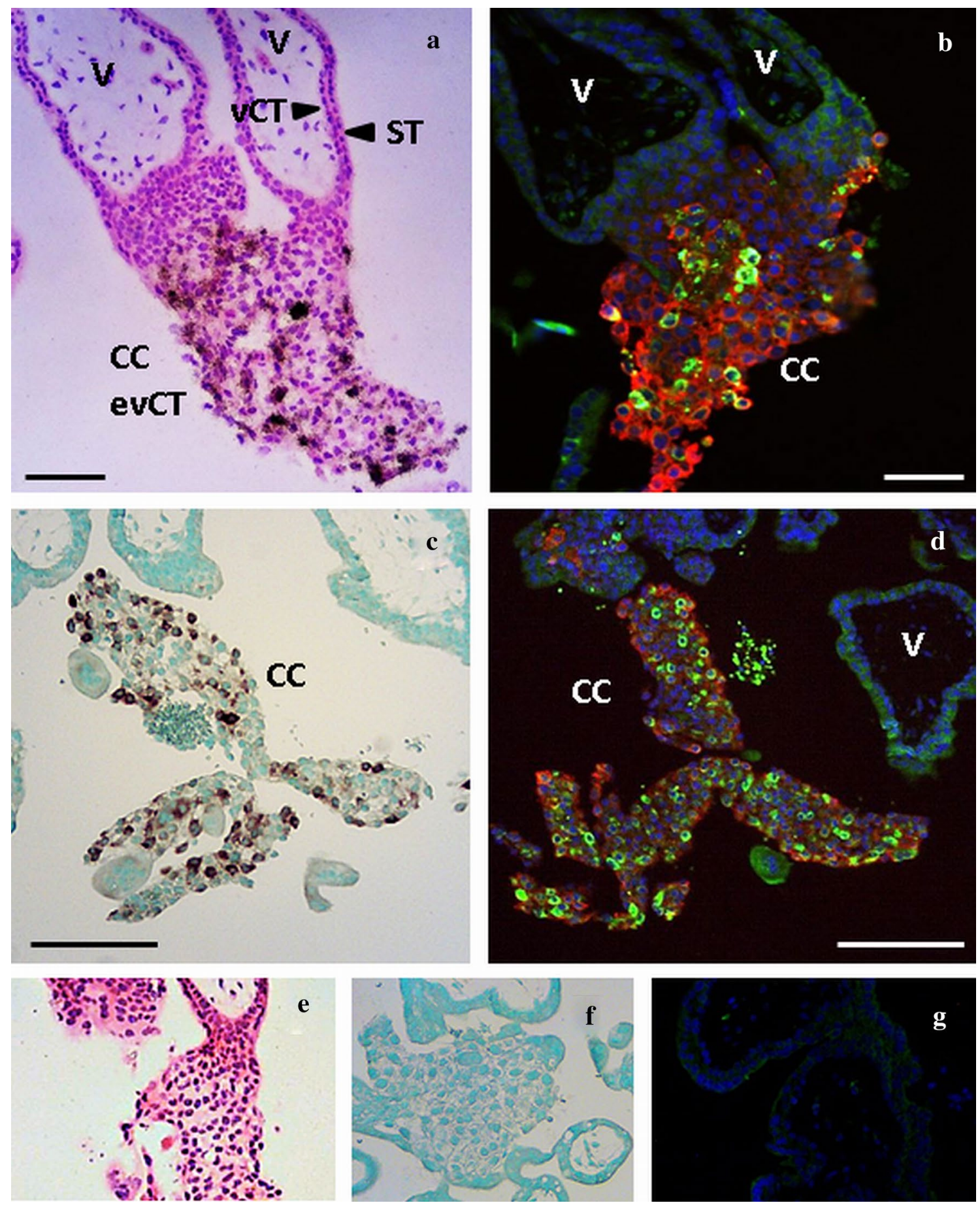

Fig. 2 Localisation of MMP12 mRNA and protein in the first trimester placenta with radioactive (a) and DIG labelled (c) in situ hybridisation and immunofluorescence (b, d). Cells stained positive for MMP12 mRNA give a black (a) or brown (c) signal in the in situ hybridisation methods. Immunofluorescence costained MMP12 (green) with HLA-G (red). $\mathbf{a}$ and $\mathbf{b}, \mathbf{c}$ and $\mathbf{d}$ represents serial sections.

decidual ECM. Both, in situ hybridisation and immunofluorescence, localised MMP12 mRNA and protein in vivo to evCT at the distal part of the cell columns and showed a-d MMP12 is located in the trophoblasts of the cell columns (CC) and absent in the placental villi (V). $S T$ syncytiotrophoblast, $v C T$ villous cytotrophoblast, evCT extravillous cytotrophoblasts. Original magnification: $\times 100$. Size of the scale bars: $100 \mu \mathrm{m}(\mathbf{a}, \mathbf{b})$ or $200 \mu \mathrm{m}$ (c, d). Negative controls for the radioactive (e), DIG labelled (f) and immunofluorescence $(\mathrm{g})$ stainings

that the number of these MMP12 producing cells declined towards the end of the first trimester. Moreover, we have demonstrated that MMP12 is upregulated under low oxygen 


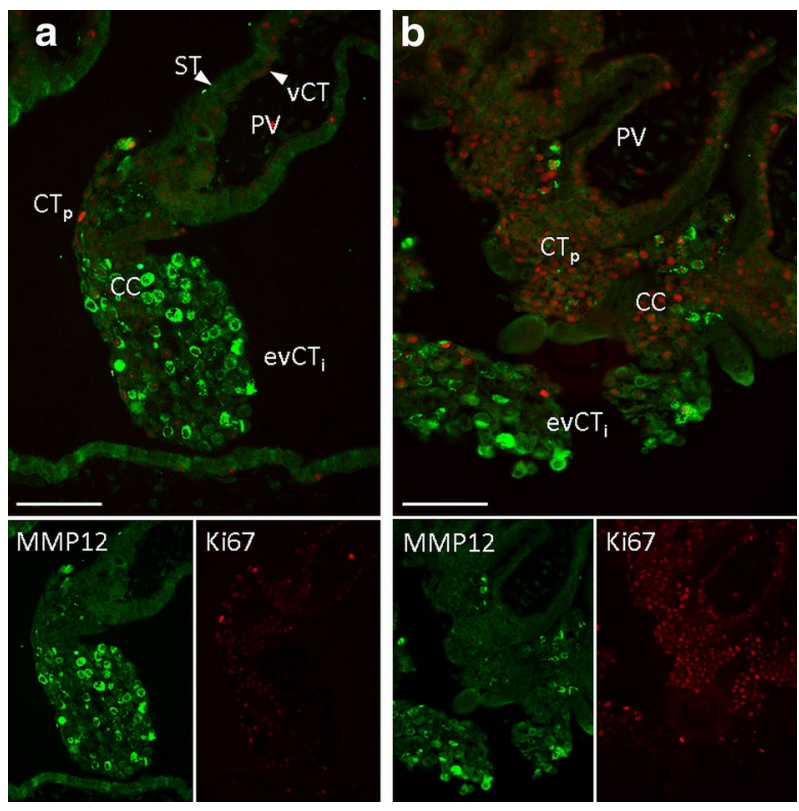

Fig. 3 Colocalisation of MMP12 (green) and the proliferation marker Ki67 (red) in the cell columns of the first trimester placenta using immunofluorescence (a, b). Ki67 antibody stained proliferating villous cytotrophoblasts ( $\mathrm{vCT}$ ) and the proliferative phenotype of the proximal part of the cell columns $\left(\mathrm{CT}_{\mathrm{p}}\right)$. MMP12 antibody predominantly stained the invasive of extravillous cytotrophoblasts $\left(\mathrm{evCT}_{\mathrm{i}}\right)$. $\mathbf{a}$ and $\mathbf{b}$ are representative images of $n=6$ different placentas. The green (MMP12) and red (Ki67) channel images are shown separately in the inserts below the overlay. $S T$ syncytiotrophoblast, $P V$ placental villus, $C C$ cell column. Original magnification: $\times 200$. Size of the scale bars: $100 \mu \mathrm{m}$

and that HIF- $1 \alpha$ activity induces MMP12 production, suggesting that the rise in oxygen at the end of the first trimester may account for this temporal regulation of MMP12.

In order to analyse the location of MMP12 we used several techniques to detect mRNA and protein in situ. Data revealed that MMP12 was expressed in a distinct evCT population, whilst it was absent in the villous cytotrophoblasts and in the syncytiotrophoblast. Concordant with previous literature, we also detected MMP12 in endovascular trophoblasts where it was previously proposed as key player in endovascular remodelling of spiral arteries due to its elastolytic activity (Harris et al. 2010). In contrast to other reports showing MMP12 mRNA or protein expression either in all trophoblast subpopulations, including villous cytotrophoblasts and syncytiotrophoblast (Harris et al. 2010), or homogeneously distributed in all trophoblasts in the cell columns (Chakraborty et al. 2016), we located MMP12 mRNA and protein expression predominantly to non-proliferating, highly invasive evCT of the distal part of the cell columns, whilst the proliferating trophoblasts of the proximal cell columns were mostly negative for MMP12.

Since MMP12 is capable of degrading various substrates present in the decidua including collagen III, fibrillin, fibronectin and laminin (Rawlings et al. 2016), MMP12 may promote decidual ECM breakdown for the invasion of evCT. Moreover, MMP12 is a potent converter of plasminogen into angiostatin (Cornelius et al. 1998). Angiostatin is an antiangiogenic molecule, has anti-proliferative effects on endothelial cells (Brauer et al. 2011) and was also shown to reduce MMP production in JEG-3 trophoblast model cells (Zhang et al. 2003). Plasmin is an alternative cleavage product of plasminogen by the plasminogen activator PLAU. Plasmin is a protease by itself, activates various MMPs (Carmeliet et al. 1997) and degrades ECM components, thus promoting invasion. Therefore, cleavage of plasmin into angiostatin by MMP12 promotes the non-invasive role of the plasminogen gene product and may represent a feedback loop to limit trophoblast MMP production, and hence, invasiveness. Thus, although a role of MMP12 in trophoblast invasion was not directly shown, its ability to degrade ECM degrading enzyme presumes such a function.

Trophoblast invasion is highest in the first trimester and declines thereafter (Hohn and Denker 2002; Shimonovitz et al. 1994). Concordantly, trophoblast production of MMPs is high during the first gestational weeks and is often attenuated already during, or after the first trimester (Xu et al. 2000). Oxygen is a key regulator of this temporal reduction of trophoblast invasion: Before the opening of uterine spiral arteries, foeto-placental development occurs in a low-oxygen environment that is thought to protect the foetus from oxidative stress. However, when maternal blood flow to the placenta starts at the beginning of the second trimester, the foeto-placental oxygen levels rise as well. In fact, low oxygen is considered as a main stimulator of trophoblast invasiveness (James et al. 2006) and the expression of various proteases lowers after the increase in placental oxygenation. For instance, some MMPs were shown to be regulated directly by HIF- $1 \alpha$, such as MMP1, MMP3 (Lin et al. 2008) and MMP14 (Wan et al. 2011).

Also the regulation of MMP12 by oxygen suggested a role of HIF- $1 \alpha$ as a classical mediator of oxygen effects. In fact, promoter analysis identified a potential HIF-1 binding site in the MMP12 promoter (Supplemental Fig. 2) and addition of DFO, a HIF- $1 \alpha$ activator, upregulated MMP12 protein. However, the effect of HIF-1 in stimulating MMP12 levels may also be mediated by wnt signalling, since $\beta$-catenin as the central molecule of wnt signalling is a target gene of HIF-1 (Liu et al. 2010) and the MMP12 promoter contains a LEF1/TCF4 binding site for wnt-induced transactivation. Moreover, the LEF1/TCF4 binding site in the MMP12 promoter provides a mechanism for gene transactivation, specifically in the non-proliferating evCT (Pollheimer et al. 2006). Transactivation of MMP12 by direct and indirect HIF-1 effects, however, may be further enhanced by activator protein 1 (AP-1) which has two binding sites in the MMP12 promoter (Yan and Boyd 2007) and can also be 
$\mathbf{a}$
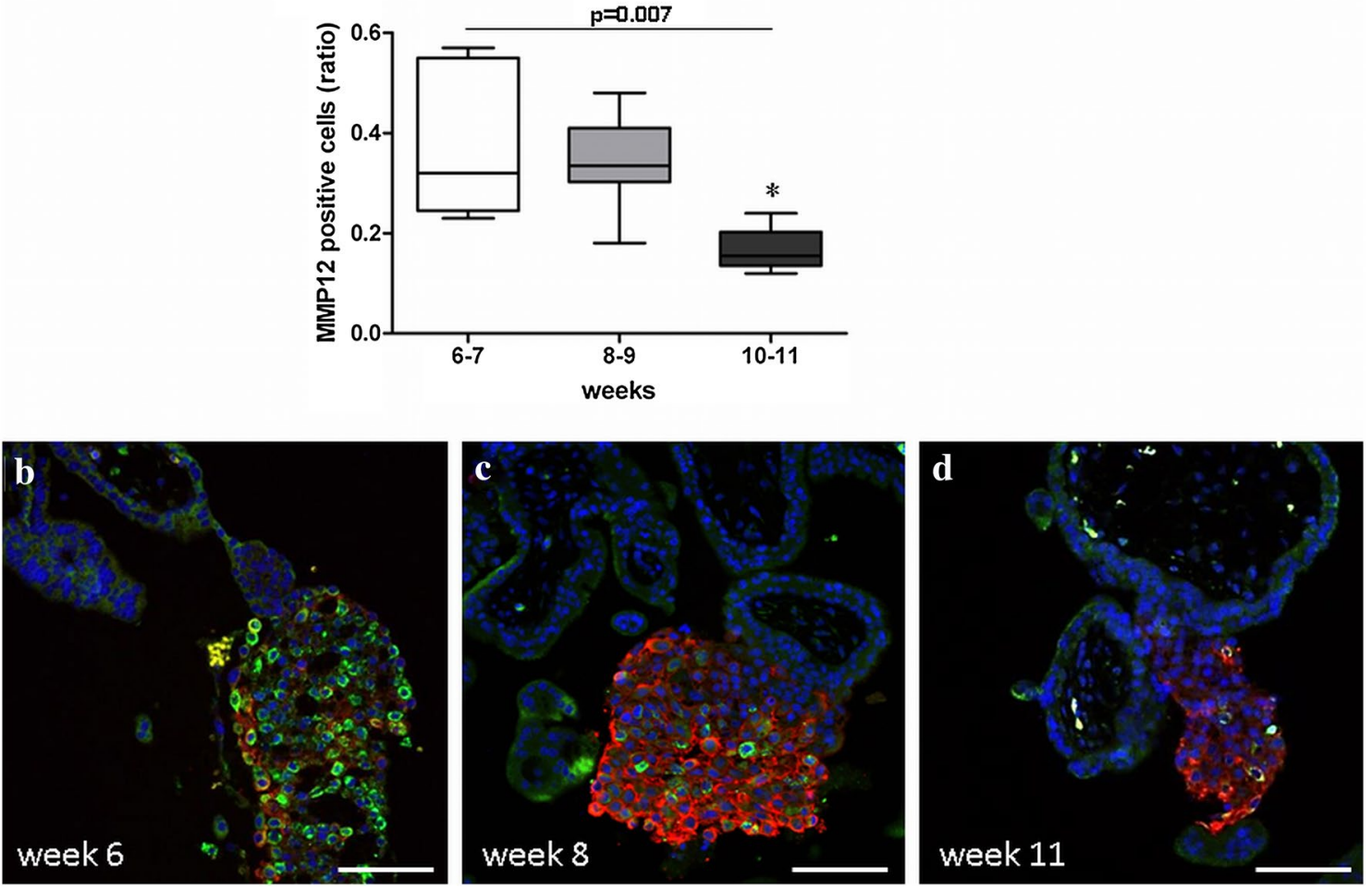

Fig. 4 Ratio of MMP12 positive cells (green) within HLA-G positive extravillous trophoblasts (red) of the cell columns throughout the first trimester of pregnancy (a). $n_{(6-7)}=5, n_{(8-9)}=8, n_{(10-11)}=6$ different tissues. Of each tissue, three sections were analysed. Statistical analysis used non-parametric ANOVA (Kruskal-Wallis). Post test (Dunn's Multiple Comparison) revealed a significant reduction in the

activated by oxidative stress (Webster et al. 1993). A potential binding site was also found for upstream transcription factor 2 (USF2), a transcription factor that was shown to regulate hypoxia responsive genes in trophoblasts (James et al. 2006). Finally, Chakraborty et al. (2016) demonstrated hypoxia-induced MMP12 expression in trophoblasts isolated from second trimester placenta (weeks 20-22), but did not assign this response to a direct effect of HIF-1, but to histone demethylation by lysyl demethylase 3A (KDM3A), which is under HIF-1 control (Wellmann et al. 2008). Indeed both, histone demethylation by KDM3A and binding of HIF-1 to its consensus sequences, may cooperate in transcriptional activation of their target genes (Mimura et al. 2012). Thus, various promoter elements in the MMP12 promoter enable MMP12 transactivation by changes in oxygen concentrations and by HIF-1 in particular, highlighting the role of oxygen in regulating MMP12 expression. Which of these possible scenarios account for our observed HIF-1 effect can only be speculated here and would require further, more detailed studies, but interplay of several pathways is likely.

In screening for invasion-relevant proteases we focused on proteases capable of degrading decidual ECM and ratio of MMP12 positive cells in weeks 10-11 (asterisk). The box plot indicates the median, 25th and 75th percentile; whiskers show minimum and maximum values. b-d Representative images of each group are shown below. Original magnification: $\times 100$. Size of the scale bars: $100 \mu \mathrm{m}$

trophoblast integrins. Proteases may also regulate activation and degradation of cytokines, growth factors and other proteases, and modulate trophoblasts in that way. Therefore, also other proteases aside from what we have selected will modulate trophoblast invasion; however, our main focus was on proteases that directly target ECM degradation.

Our screening approach to identify invasion-relevant proteases revealed MMP12 expression levels in FT even higher than levels of $M M P 2$ and $M M P 9$, the two most investigated proteases in trophoblast invasion, which were also identified as invasion relevant by our screening. Moreover, we also found several other MMPs and ADAMs, highlighting the role of these two metalloprotease families in pregnancy establishment. Also PLAU has earlier been implicated in invading trophoblast invasion (Anteby et al. 2004). Two proteases, however, have not yet been assigned to trophoblast invasion, i.e. PCSK6 and PCSK7. Both enzymes possess a broad range of substrates, including integrins, fibrillin and various MMPs and ADAMs (MEROPS database). In fact, PCSK6 was implicated in cancer invasion (Wang et al. 2015), a process that resembles trophoblast invasion in many instances. 
Fig. 5 Effect of oxygen on MMP12 protein in primary first trimester trophoblasts. Isolated trophoblasts were cultured at 5,12 and $21 \%$ oxygen for $48 \mathrm{~h}$. A representative immunoblot is shown on top (a). Quantified data are given as mean \pm SEM (b). $n=7$ different trophoblast isolations between weeks 7 and 11 , each in duplicate. Statistical analysis used non-parametric ANOVA (Kruskal-Wallis). Post test (Dunn's Multiple Comparison) revealed a significant reduction in MMP12 protein at 12 and $21 \%$ oxygen when compared to $5 \%$ oxygen (asterisk), but oxygen effect was abrogated when the HIF- $1 \alpha$ activator DFO was added $\mathbf{a}$

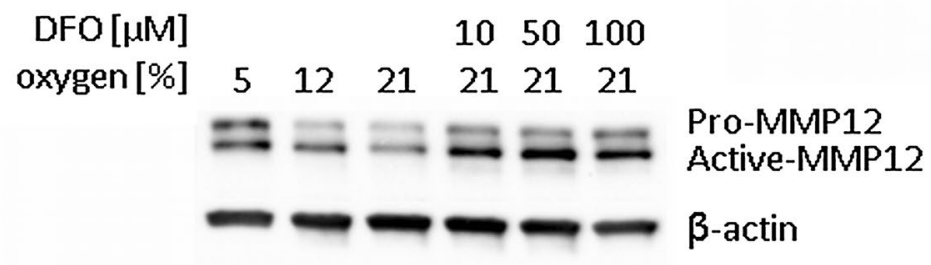

b

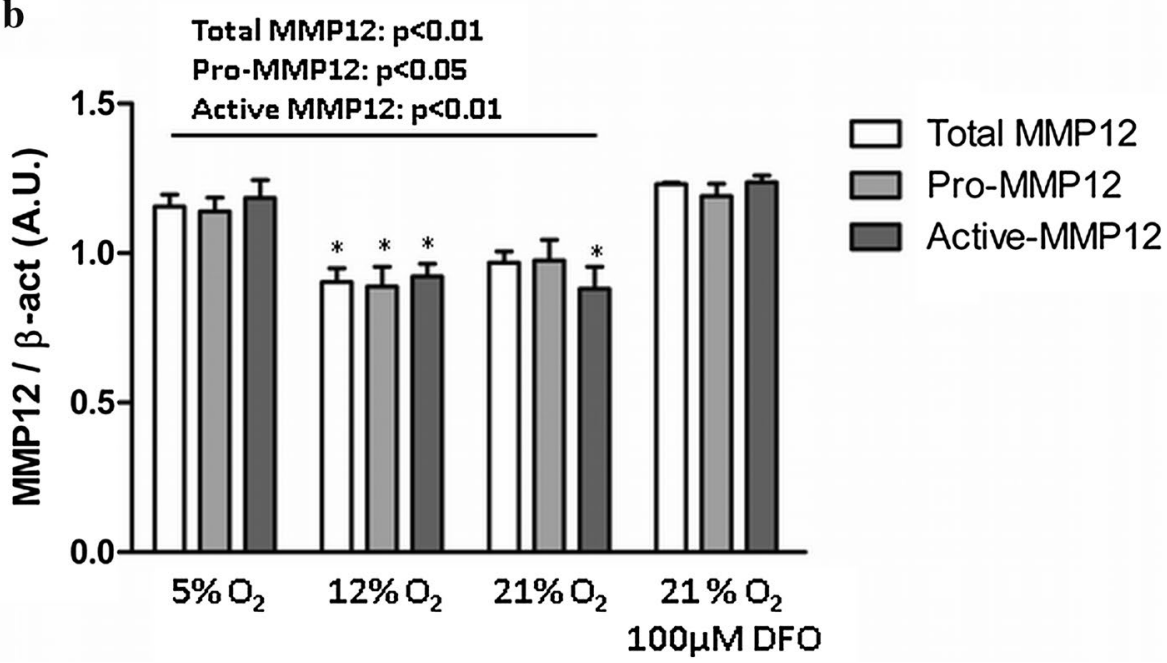

In this study, we used primary FT and TT as models for invasive and non-invasive trophoblast phenotypes, respectively. Although these models are primary cells, we are aware that taken out of their physiological environment and cultured in vitro, they are exposed to artificial environment which may alter their functionality or differentiation status. However, cultured FT isolated by our method are HLA-G positive (Ghaffari-Tabrizi-Wizsy et al. 2014) and perform in vitro invasion (Majali-Martinez et al. 2017), whilst cultured TT represent a HLA-G negative trophoblast population able to differentiate and fuse into syncytial structures (Simpson et al. 1992). Therefore, we regard these cells as an appropriate model for our screening approach.

The screening approach for invasion-relevant proteases was limited by the fact that FT were cultured at ambient oxygen levels, as in vivo FT are exposed to low-oxygen environment. However, the experiments measuring the effect of oxygen on MMP12 levels in FT revealed that the distinct expression of MMP12 in FT vs TT by far exceeded this effect of oxygen.

We have shown that MMP12 is specifically expressed by non-proliferating invasive trophoblasts of the trophoblast cell columns and that MMP12 expressing cells decrease at the end of the first trimester. Furthermore, we have demonstrated oxygen as a key regulator for MMP12 expression and proved HIF-1 to induce MMP12. This may occur either directly via the potential HIF-1 binding site in the MMP12 promoter or indirectly via upregulation of other transcriptional regulators by HIF-1. Therefore, this study implies that MMP12 may represent a key protease in trophoblast invasion during first trimester pregnancy.

Acknowledgements Open access funding provided by Medical University of Graz.

\section{Compliance with ethical standards}

Conflict of interest The authors declare they have no conflict of interest.

Open Access This article is distributed under the terms of the Creative Commons Attribution 4.0 International License (http://creativecommons.org/licenses/by/4.0/), which permits unrestricted use, distribution, and reproduction in any medium, provided you give appropriate credit to the original author(s) and the source, provide a link to the Creative Commons license, and indicate if changes were made.

\section{References}

Anacker J, Segerer SE, Hagemann C, Feix S, Kapp M, Bausch R, Kammerer U (2011) Human decidua and invasive trophoblasts 
are rich sources of nearly all human matrix metalloproteinases. Mol Hum Reprod 17(10):637-652. doi:10.1093/molehr/gar033

Anteby EY, Greenfield C, Natanson-Yaron S, Goldman-Wohl D, Hamani Y, Khudyak V, Ariel I, Yagel S (2004) Vascular endothelial growth factor, epidermal growth factor and fibroblast growth factor- 4 and -10 stimulate trophoblast plasminogen activator system and metalloproteinase-9. Mol Hum Reprod 10(4):229-235. doi:10.1093/molehr/gah031

Aplin JD, Haigh T, Lacey H, Chen CP, Jones CJ (2000) Tissue interactions in the control of trophoblast invasion. J Reprod Fertil Suppl 55:57-64

Bischof P, Irminger-Finger I (2005) The human cytotrophoblastic cell, a mononuclear chameleon. Int J Biochem Cell Biol 37(1):1-16. doi:10.1016/j.biocel.2004.05.014

Blaschitz A, Weiss U, Dohr G, Desoye G (2000) Antibody reaction patterns in first trimester placenta: implications for trophoblast isolation and purity screening. Placenta 21(7):733-741. doi:10.1053/ plac.2000.0559

Brauer R, Beck IM, Roderfeld M, Roeb E, Sedlacek R (2011) Matrix metalloproteinase-19 inhibits growth of endothelial cells by generating angiostatin-like fragments from plasminogen. BMC Biochem 12:38. doi:10.1186/1471-2091-12-38

Carmeliet P, Moons L, Lijnen R, Baes M, Lemaitre V, Tipping P, Drew A, Eeckhout Y, Shapiro S, Lupu F, Collen D (1997) Urokinasegenerated plasmin activates matrix metalloproteinases during aneurysm formation. Nat Genet 17(4):439-444. doi:10.1038/ ng 1297-439

Cervar M, Blaschitz A, Dohr G, Desoye G (1999) Paracrine regulation of distinct trophoblast functions in vitro by placental macrophages. Cell Tissue Res 295(2):297-305

Chakraborty D, Cui W, Rosario GX, Scott RL, Dhakal P, Renaud SJ, Tachibana M, Rumi MA, Mason CW, Krieg AJ, Soares MJ (2016) HIF-KDM3A-MMP12 regulatory circuit ensures trophoblast plasticity and placental adaptations to hypoxia. Proc Natl Acad Sci USA 113(46):E7212-E7221. doi:10.1073/pnas.1612626113

Cohen M, Meisser A, Bischof P (2006) Metalloproteinases and human placental invasiveness. Placenta 27(8):783-793. doi:10.1016/j. placenta.2005.08.006

Cornelius LA, Nehring LC, Harding E, Bolanowski M, Welgus HG, Kobayashi DK, Pierce RA, Shapiro SD (1998) Matrix metalloproteinases generate angiostatin: effects on neovascularization. $\mathrm{J}$ Immunol 161(12):6845-6852

Farre D, Roset R, Huerta M, Adsuara JE, Rosello L, Alba MM, Messeguer X (2003) Identification of patterns in biological sequences at the ALGGEN server: PROMO and MALGEN. Nucleic Acids Res 31(13):3651-3653

Fekry B, Jeffries KA, Esmaeilniakooshkghazi A, Ogretmen B, Krupenko SA, Krupenko NI (2016) CerS6 is a novel transcriptional target of $\mathrm{p} 53$ protein activated by non-genotoxic stress. J Biol Chem 291(32):16586-16596. doi:10.1074/jbc.M116.716902

Ghaffari-Tabrizi-Wizsy N, Cvitic S, Tam-Amersdorfer C, Bilban M, Majali-Martinez A, Schramke K, Desoye G, Hiden U (2014) Different preference of degradome in invasion versus angiogenesis. Cells Tissues Organs 200(3-4):181-194. doi:10.1159/000381766

Harris LK, Smith SD, Keogh RJ, Jones RL, Baker PN, Knofler M, Cartwright JE, Whitley GS, Aplin JD (2010) Trophoblast- and vascular smooth muscle cell-derived MMP-12 mediates elastolysis during uterine spiral artery remodeling. Am J Pathol 177(4):2103-2115. doi:10.2353/ajpath.2010.100182

Hohn HP, Denker HW (2002) Experimental modulation of cell-cell adhesion, invasiveness and differentiation in trophoblast cells. Cells Tissues Organs 172(3):218-236. doi:10.1159/000066965

Huppertz B, Gauster M, Orendi K, Konig J, Moser G (2009) Oxygen as modulator of trophoblast invasion. J Anat 215(1):14-20. doi:10.1111/j.1469-7580.2008.01036.x
James JL, Stone PR, Chamley LW (2006) The regulation of trophoblast differentiation by oxygen in the first trimester of pregnancy. Hum Reprod Update 12(2):137-144. doi:10.1093/humupd/dmi043

Jauniaux E, Watson AL, Hempstock J, Bao YP, Skepper JN, Burton GJ (2000) Onset of maternal arterial blood flow and placental oxidative stress. A possible factor in human early pregnancy failure. Am J Pathol 157(6):2111-2122. doi:10.1016/S0002-9440(10)64849-3

Kaufmann P, Black S, Huppertz B (2003) Endovascular trophoblast invasion: implications for the pathogenesis of intrauterine growth retardation and preeclampsia. Biol Reprod 69(1):1-7. doi:10.1095/biolreprod.102.014977

Kemp B, Kertschanska S, Kadyrov M, Rath W, Kaufmann P, Huppertz B (2002) Invasive depth of extravillous trophoblast correlates with cellular phenotype: a comparison of intra- and extrauterine implantation sites. Histochem Cell Biol 117(5):401-414. doi:10.1007/s00418-002-0396-0

Lin JL, Wang MJ, Lee D, Liang CC, Lin S (2008) Hypoxia-inducible factor-1alpha regulates matrix metalloproteinase- 1 activity in human bone marrow-derived mesenchymal stem cells. FEBS Lett 582(17):2615-2619. doi:10.1016/j.febslet.2008.06.033

Liu L, Zhu XD, Wang WQ, Shen Y, Qin Y, Ren ZG, Sun HC, Tang ZY (2010) Activation of beta-catenin by hypoxia in hepatocellular carcinoma contributes to enhanced metastatic potential and poor prognosis. Clin Cancer Res 16(10):2740-2750. doi:10.1158/10780432.CCR-09-2610

Majali-Martinez A, Velicky P, Pollheimer J, Knofler M, Yung HW, Burton GJ, Tabrizi-Wizsy NG, Lang U, Hiden U, Desoye G, Dieber-Rotheneder M (2017) Endothelin-1 down-regulates matrix metalloproteinase 14 and 15 expression in human first trimester trophoblasts via endothelin receptor type B. Hum Reprod 32(1):46-54. doi:10.1093/humrep/dew295

Messeguer X, Escudero R, Farre D, Nunez O, Martinez J, Alba MM (2002) PROMO: detection of known transcription regulatory elements using species-tailored searches. Bioinformatics 18(2):333-334

Mimura I, Nangaku M, Kanki Y, Tsutsumi S, Inoue T, Kohro T, Yamamoto S, Fujita T, Shimamura T, Suehiro J, Taguchi A, Kobayashi M, Tanimura K, Inagaki T, Tanaka T, Hamakubo T, Sakai J, Aburatani H, Kodama T, Wada Y (2012) Dynamic change of chromatin conformation in response to hypoxia enhances the expression of GLUT3 (SLC2A3) by cooperative interaction of hypoxiainducible factor 1 and KDM3A. Mol Cell Biol 32(15):3018-3032. doi:10.1128/MCB.06643-11

Moser G, Huppertz B (2017) Implantation and extravillous trophoblast invasion: from rare archival specimens to modern biobanking. Placenta. doi:10.1016/j.placenta.2017.02.007

Moser G, Orendi K, Gauster M, Siwetz M, Helige C, Huppertz B (2011) The art of identification of extravillous trophoblast. Placenta 32(2):197-199. doi:10.1016/j.placenta.2010.11.008

Oefner CM, Sharkey A, Gardner L, Critchley H, Oyen M, Moffett A (2015) Collagen type IV at the fetal-maternal interface. Placenta 36(1):59-68. doi:10.1016/j.placenta.2014.10.012

Pollheimer J, Loregger T, Sonderegger S, Saleh L, Bauer S, Bilban M, Czerwenka K, Husslein P, Knofler M (2006) Activation of the canonical wingless/T-cell factor signaling pathway promotes invasive differentiation of human trophoblast. Am J Pathol 168(4):1134-1147. doi:10.2353/ajpath.2006.050686

Pollheimer J, Fock V, Knofler M (2014) Review: the ADAM metalloproteinases-novel regulators of trophoblast invasion? Placenta 35(Suppl):S57-S63. doi:10.1016/j.placenta.2013.10.012

Polliotti BM, Abramowsky C, Schwartz DA, Keesling SS, Lee GR, Caba J, Zhang W, Panigel M, Nahmias AJ (1995) Culture of firsttrimester and full-term human chorionic villus explants: role of human chorionic gonadotropin and human placental lactogen as a viability index. Early Pregnancy 1(4):270-280 
Pringle KG, Kind KL, Sferruzzi-Perri AN, Thompson JG, Roberts CT (2010) Beyond oxygen: complex regulation and activity of hypoxia inducible factors in pregnancy. Hum Reprod Update 16(4):415-431. doi:10.1093/humupd/dmp046

Rawlings ND, Barrett AJ, Finn R (2016) Twenty years of the MEROPS database of proteolytic enzymes, their substrates and inhibitors. Nucleic Acids Res 44(D1):D343-D350. doi:10.1093/nar/gkv1118

Salceda S, Caro J (1997) Hypoxia-inducible factor 1alpha (HIF-1alpha) protein is rapidly degraded by the ubiquitin-proteasome system under normoxic conditions. Its stabilization by hypoxia depends on redox-induced changes. J Biol Chem 272(36):22642-22647

Shimonovitz S, Hurwitz A, Dushnik M, Anteby E, Geva-Eldar T, Yagel S (1994) Developmental regulation of the expression of 72 and $92 \mathrm{kd}$ type IV collagenases in human trophoblasts: a possible mechanism for control of trophoblast invasion. Am J Obstet Gynecol 171(3):832-838

Simpson RA, Mayhew TM, Barnes PR (1992) From 13 weeks to term, the trophoblast of human placenta grows by the continuous recruitment of new proliferative units: a study of nuclear number using the disector. Placenta 13(5):501-512

Wan J, Chai H, Yu Z, Ge W, Kang N, Xia W, Che Y (2011) HIF1alpha effects on angiogenic potential in human small cell lung carcinoma. J Exp Clin Cancer Res CR 30:77. doi:10.1186/1756-9966-30-77

Wang F, Wang L, Pan J (2015) PACE4 regulates proliferation, migration and invasion in human breast cancer MDA-MB-231 cells. Mol Med Rep 11(1):698-704. doi:10.3892/mmr.2014.2691

Webster KA, Discher DJ, Bishopric NH (1993) Induction and nuclear accumulation of fos and jun proto-oncogenes in hypoxic cardiac myocytes. J Biol Chem 268(22):16852-16858

Wellmann S, Bettkober M, Zelmer A, Seeger K, Faigle M, Eltzschig HK, Buhrer C (2008) Hypoxia upregulates the histone demethylase JMJD1A via HIF-1. Biochem Biophys Res Commun 372(4):892-897. doi:10.1016/j.bbrc.2008.05.150

Xu P, Wang YL, Zhu SJ, Luo SY, Piao YS, Zhuang LZ (2000) Expression of matrix metalloproteinase-2, -9 , and -14 , tissue inhibitors of metalloproteinase-1, and matrix proteins in human placenta during the first trimester. Biol Reprod 62(4):988-994

Yan C, Boyd DD (2007) Regulation of matrix metalloproteinase gene expression. J Cell Physiol 211(1):19-26. doi:10.1002/jcp.20948

Zhang J, Cao YJ, Li FY, Li J, Yao LB, Duan EK (2003) Effects of fibronectin, VEGF and angiostatin on the expression of MMPs through different signaling pathways in the JEG-3 cells. Am J Reprod Immunol 50(4):273-285 